\title{
QUANTIFICATION OF STRESS BY LEVELS ON TREAD MILL EXERCISE
}

\author{
R. Karunadevi, Ganesh T. Waghmode
}

1. Associate Professor. Department of Physiology, NRI Institute of Medical Sciences. Sangivalasa, Vishakhapatnam, Andhra Pradesh, India.

2. Associate Professor. Department of Anatomy, NRI Institute of Medical Sciences. Sangivalasa, Vishakhapatnam, Andhra Pradesh, India.

\section{CORRESPONDING AUTHOR:}

Dr.R. Karunadevi, D/No.49/9/33,lalithanagar,

Near sangam office bus stand,

Vishakhapatanam, Pin-530016.

E-mail: karunarednam@yahoo.com

ABSTRACT: The present study is carried out with the objective of quantifying the physical stress of exercise on Treadmill, in group healthy medical students, using established methodology. After recording the basic physical data every subject exercised on Treadmill on the lines of Bruce Protocol till the point of exhaustion. Duration of exercise sustained and METs are recorded for each subject. From the values of body mass, angle of inclination, belt speed, and certain constants, work output for the exercise period in Joules and Kilocalories are derived using appropriate standard formulae and tabulated.

Above study gives information that:

A linear relation between duration of exercise and METs is established.

To eliminate the influence of body mass variations among individuals, energy cost is better expressed as $\mathrm{Kcal} / \mathrm{Kg} / \mathrm{min}$.

There is no universal agreement about the predictive value of METs as quantitative index of 02 consumption due to its inherent short coming discussed.

To facilitate this it is opined that, computer programmers of Treadmill stress test should incorporate appropriate software so to generate energy cost values in terms of Kilocalories, in addition to the METs currently practiced.

One should appreciate that the utility value of quantification of exercise stress is enormous. It provides a scientific basis to access the absolute quantity of work an individual can withstand on Treadmill and to plan suitable dietary regimens, weight reduction programmes and to design ways of optimizing work rates and skilled development and achieve economy of energy expenditure for a given task.

KEY WORDS: Metabolic equivalent, energy expenditure, point of exhaustion, heart rate, blood pressure and hemoglobin.

MESH TERM: Heart rate[E01-370-600-835-500],Blood pressure (arterial)-[G0-330-380

076-347], Hemoglobin-[D12-776-432-316-762] and Exercise-[E01-370-386-700-250]

INTRODUCTION: The present study approaches the evaluation of stress from a new dimension i.e., by actually attempting to quantitate the physical work involved (in joules or its equivalent energy expenditure in Kcals) in any given stage of treadmill exercise. A knowledge of such quantitative dimensions of stress during treadmill exercise provides better insight into physiology of stress such studies also ascertain any correlation between work output and certain physiological variables like body mass, Hemoglobin and duration of exercise. 
One should appreciate that the utility value of quantification of exercise stress is enormous. It provides a scientific basis to access the absolute quantity of work an individual can withstand on treadmill and to suitable dietary regimens, weight reduction programmes, and to design ways of optimizing work rates and skilled development and achieve economy of energy expenditure for a given task.

MATERIALS AND METHODS: Due to easy accessibility the present study was carried out in a group of 20 healthy $1^{\text {st }}$ year male medical students who are not regular athletes. Their consent was taken. The study was carried in Rangaraya Medical Collage, Kakinada.

Individual Hemoglobin content were estimated. The test procedure was carried out in each individual till the point of exhaustion based on subjective/objective criteria. The duration of exercise (in min) and Mets were also recorded.

The Bruce multistage treadmill protocol is followed in the present study. It has the advantage of progressively increasing the exercise load, beginning with a stage low enough to be tolerated by the weakest candidate and with a stage sufficiently difficult to challenge the 'fittest candidate'. Each stage is long enough in duration for the subject to reach or closely approach steady state. It begins at $2.7 \mathrm{Kmph}$ and $10 \%$ grade, a level of approximately 5 METS with increments in speed and grade every 3 minutes that allow total body aerobic work to increase close to one MET for every minute of exercise. The 3 stages allow approximate equilibrium to be attained by the end of each stage, so that the test results can be recorded in the last minute of each stage. The test provides information regarding the functional aerobic capacity of the subject. The table below presents the salient of Bruce protocol of treadmill exercise testing.

BRUCE PROTOCOL

\begin{tabular}{|l|l|l|l|l|l|l|}
\hline $\begin{array}{l}\text { Stage } \\
\text { Number }\end{array}$ & $\begin{array}{l}\text { Time } \\
\text { required for } \\
\text { Stage(min) }\end{array}$ & $\begin{array}{l}\text { Elapsed } \\
\text { time at the } \\
\text { end of } \\
\text { stage(min) }\end{array}$ & $\begin{array}{l}\text { Speed } \\
\text { Kmph }\end{array}$ & Grades\% & $\begin{array}{l}\text { Elevation } \\
\text { (degrees) }\end{array}$ & METS \\
\hline 1 & 3 & 3 & 2.7 & 10 & 5.7 & 5 \\
\hline 2 & 3 & 6 & 4.0 & 12 & 6.8 & 7 \\
\hline 3 & 3 & 9 & 5.5 & 14 & 8.0 & 10 \\
\hline 4 & 3 & 12 & 6.8 & 16 & 9.0 & 14 \\
\hline 5 & 3 & 15 & 8.0 & 18 & 10.0 & 16.3 \\
\hline 6 & 3 & 18 & 8.9 & 20 & 11 & 19.5 \\
\hline 7 & 3 & 21 & 9.6 & 22 & 12.4 & 22.7 \\
\hline
\end{tabular}

METS: Exercise capacity is often reported in terms of "Metabolic Equivalets" or METs, in lieu of V02 max. Rather than determining each person's true resting oxygen uptake. The unit of metabolic equivalent, MET is widely employed and the same is followed in the present study. The MET is a unit of sitting/ resting oxygen uptake ( $3.5 \mathrm{ml} 02$ per Kg body weight per minute). The oxygen uptake during exercise is conveniently expressed in multiples of sitting / resting requirements (METs). The treadmill system used in the present study gives values of METs at each grade of activity

The length of time each individual could exercise on the treadmill is recorded for comparison of endurance. 
QUANTITATING EFFORT: During treadmill exercise, individual runs on treadmill belt and advances horizontally and in the process also lifts the body at certain inclination. The treadmill belt velocity and inclination were changed with each 3 minute phase of Bruce protocol.

External work during inclined treadmill exercise can be calculated from the sum of horizontal velocity $(\mathrm{V} \cos \theta)$ and vertical velocity $(\mathrm{V} \sin \theta)$ i.e., the sum of horizontal components of work (used to move the body horizontal direction and the vertical direction component of work used to move in the vertical direction).

From the values of body weight, Belt friction $(\mu)$, treadmill angle (inclination) and velocity of belt $(\mathrm{V})$ at each stage of the protocol, as well as exercise duration the work done is calculated using mathematical formulae as given below. Bruce protocol specifies the treadmill angle, velocity of belt $(\mathrm{V})$ and duration of exercise for each phase as detailed already in this section.

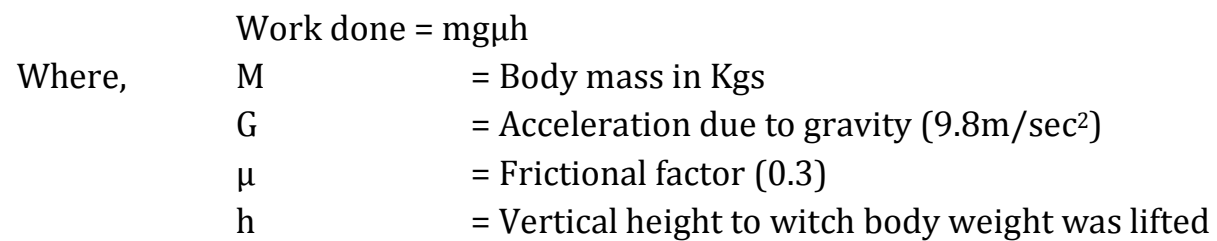

' $h$ ' is calculated from the product of horizontal movement and vertical movement $(\cos \theta+\sin \theta)$ and treadmill belt speed(V) and exercise duration $t$.

$$
\text { i.e., }(\cos \theta+\sin \theta) v t \text {. }
$$

Therefore work done in joules $/ \sec =m g(\mu \cos \theta+\sin \theta) v t$.

STATISTICAL METHODS: The arithmetical mean, STANDARD DEVIATION and STANDARD ERROR was calculated for each parameter. 
TABLE - I

\begin{tabular}{|c|c|c|c|c|c|c|c|c|c|c|c|}
\hline \multicolumn{12}{|c|}{ TABLE - I } \\
\hline \multicolumn{12}{|c|}{ The stage wise Energy Expenditure and METS } \\
\hline & \multirow{3}{*}{$\begin{array}{l}\text { Body } \\
\text { Mass } \\
\text { in Kgs }\end{array}$} & \multicolumn{4}{|c|}{ Treadmill exercise Progression Stages } & \multirow{3}{*}{$\begin{array}{c}\text { Total } \\
\text { Duration } \\
\text { of } \\
\text { Exercise }\end{array}$} & \multirow{2}{*}{\multicolumn{2}{|c|}{$\begin{array}{c}\text { Total Energy } \\
\text { Expenditure during } \\
\text { Exercise Period }\end{array}$}} & \multirow{3}{*}{ Mets } & \multirow{3}{*}{$\begin{array}{c}\text { Average } \\
\text { Rate of } \\
\text { Energy } \\
\text { Expended } \\
\text { during } \\
\text { Exercise } \\
\text { Period } \\
\text { Kcal/min }\end{array}$} & \multirow{3}{*}{$\begin{array}{c}\text { Energy } \\
\text { Expend } \\
\text { iture } \\
\text { for Kg } \\
\text { Body } \\
\text { Weight } \\
\text { per } \\
\text { min }\end{array}$} \\
\hline & & $\begin{array}{l}\text { Stage -I } \\
\text { Speed } \\
2.7 \\
\text { KMPH } \\
\text { Angle } \\
5.7^{\circ} \mathrm{t}- \\
3 \mathrm{~min} \\
\text { Mets-5 }\end{array}$ & $\begin{array}{l}\text { Stage -II } \\
\text { Speed } 4 \\
\text { KMPH } \\
\text { Angle } 6.8^{\circ} \\
\text { Elapsed } \\
\text { time-6min } \\
\text { t-3min } \\
\text { Mets-7 }\end{array}$ & $\begin{array}{l}\text { Stage -III } \\
\text { Speed } 5.5 \\
\text { KMPH } \\
\text { Angle } 8^{\circ} \\
\text { Elapsed } \\
\text { time- } \\
9 \text { min t- } \\
\text { 3min } \\
\text { Mets-10 }\end{array}$ & $\begin{array}{l}\text { Stage -IV } \\
\text { Speed } 6.8 \\
\text { KMPH } \\
\text { Angle 9॰ } \\
\text { Elapsed } \\
\text { time- } \\
12 \mathrm{~min} \text { t- } \\
\text { 3min } \\
\text { Mets-14 }\end{array}$ & & & & & & \\
\hline S.No & & \multicolumn{4}{|c|}{ Energy expenditure in Kcal in each phase } & & Joules & Kcal & & & \\
\hline 1 & 68 & 8.56 & 13.27 & 19.12 & 23.17 & 11.80 & 267223.27 & 64.11 & 13.70 & 5.42 & 0.08 \\
\hline 2 & 68 & 8.56 & 13.27 & 7.22 & & 7.13 & 121078.15 & 29.05 & 8.10 & 4.07 & 0.06 \\
\hline 3 & 45 & 5.66 & 8.78 & 1.31 & & 6.31 & 65652.99 & 15.75 & 7.30 & 2.5 & 0.06 \\
\hline 4 & 47 & 5.92 & 9.17 & 13.21 & 0.79 & 9.14 & 121254.09 & 29.09 & 10.20 & 3.18 & 0.07 \\
\hline 5 & 47 & 5.92 & 9.17 & 1.15 & & 6.26 & 67652.96 & 16.23 & 7.20 & 2.59 & 0.06 \\
\hline 6 & 47 & 5.92 & 9.17 & 13.21 & 2.83 & 9.5 & 129734.27 & 31.13 & 10.60 & 3.28 & 0.07 \\
\hline 7 & 48 & 6.04 & 9.37 & 13.50 & 0.06 & 9.01 & 120706.52 & 28.96 & 10.00 & 3.21 & 0.07 \\
\hline 8 & 48 & 6.04 & 9.37 & 5.76 & & 7.28 & 88216.85 & 21.17 & 8.17 & 2.91 & 0.06 \\
\hline 9 & 48 & 6.04 & 9.37 & 13.50 & 1.10 & 9.19 & 125036.83 & 30.00 & 10.20 & 3.26 & 0.07 \\
\hline 10 & 48 & 6.04 & 9.37 & 5.31 & & 7.18 & 86341.90 & 20.72 & 8.10 & 3.89 & 0.06 \\
\hline 11 & 49 & 6.17 & 9.56 & 13.78 & 3.48 & 9.59 & 137465.14 & 32.98 & 10.70 & 3.44 & 0.07 \\
\hline 12 & 49 & 6.17 & 9.56 & 1.56 & & 6.34 & 72063.02 & 17.29 & 7.30 & 2.73 & 0.06 \\
\hline 13 & 45 & 5.66 & 8.78 & 0.13 & & 6.01 & 60748.83 & 14.58 & 7.00 & 2.42 & 0.05 \\
\hline 14 & 64 & 8.06 & 12.49 & 17.99 & 23.09 & 12.00 & 256850.31 & 61.62 & 14.00 & 5.14 & 0.08 \\
\hline 15 & 71 & 8.94 & 13.85 & 13.31 & & 8.00 & 150455.61 & 36.10 & 9.00 & 4.51 & 0.06 \\
\hline 16 & 80 & 10.07 & 15.61 & & & 6.00 & 107029.20 & 25.68 & 7.00 & 4.28 & 0.05 \\
\hline 17 & 72 & 9.06 & 14.05 & 20.24 & & 9.00 & 180968.92 & 43.35 & 10.00 & 4.82 & 0.07 \\
\hline 18 & 61 & 7.68 & 11.9 & 17.15 & 22.01 & 12.00 & 244810.45 & 58.74 & 14.00 & 4.89 & 0.08 \\
\hline 19 & 69 & 8.68 & 13.46 & 19.40 & 21.57 & 11.60 & 263083.81 & 63.12 & 13.40 & 5.44 & 0.08 \\
\hline 20 & 54 & 6.8 & 10.54 & 7.59 & & 7.50 & 103884.45 & 24.92 & 8.50 & 3.32 & 0.06 \\
\hline Mean & 57.98 & 7.30 & 11.31 & 15.58 & 9.95 & 9.32 & $160,133.47$ & 38.47 & 10.57 & 4.00 & 0.07 \\
\hline $\begin{array}{l}\mathrm{SD}(+/ \\
-)\end{array}$ & 9.66 & 1.20 & 1.86 & 9.99 & 9.16 & 1.86 & $59,265.57$ & 14.22 & 2.22 & 0.86 & 0.01 \\
\hline SE & 1.53 & 0.19 & 0.29 & 1.58 & 1.45 & 0.29 & $9,370.71$ & 2.25 & 0.35 & 0.14 & 0.00 \\
\hline
\end{tabular}




\begin{tabular}{|r|r|r|r|r|}
\hline \multicolumn{7}{|c|}{ TABLE-II } \\
\multicolumn{7}{|r|}{ COMPARITIVE DATA PHYSIQUE, HB\%, DURATION OF EXERCISE AND ENERGY } \\
COST IN KCAL AND METS
\end{tabular}


Table - III

\begin{tabular}{|c|c|c|c|c|c|c|c|c|}
\hline & \multirow{2}{*}{$\begin{array}{l}\text { Duration } \\
\text { exircise }\end{array}$} & & \multirow{2}{*}{$\begin{array}{l}\text { Phase- } \\
\text { I 3min }\end{array}$} & \multirow{2}{*}{$\begin{array}{l}\text { Phase - } \\
\text { II } \\
6 \mathrm{~min}\end{array}$} & \multicolumn{2}{|l|}{$\begin{array}{l}\text { Phase-III } \\
9 \mathrm{~min}\end{array}$} & \multicolumn{2}{|l|}{$\begin{array}{l}\text { Phase-IV } \\
12 \mathrm{~min}\end{array}$} \\
\hline & & & & & $\begin{array}{l}\text { Dis } \\
\text { Continued }\end{array}$ & Completed & $\begin{array}{l}\text { Dis } \\
\text { Continued }\end{array}$ & Completed \\
\hline & Velocity in KMPH & 1 & 2.7 & 4.00 & 5.50 & 5.50 & 6.80 & 6.80 \\
\hline & Inclination & 2 & 5.70 & 6.80 & 8.00 & 3.00 & 9.00 & 9.00 \\
\hline \multirow{5}{*}{ 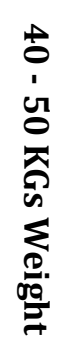 } & Time & 3 & 3.00 & 6.00 & 6.56 & 9.00 & 9.41 & 12.00 \\
\hline & $\mathbf{n}$ & 4 & 15 & 15 & 6 & 9 & 8 & 1 \\
\hline & K Cal & 5 & 6.01 & 15.32 & 17.86 & 31.42 & 33.80 & 52.20 \\
\hline & K Cal/ min & 6 & 2.00 & 2.50 & 2.72 & 3.49 & 3.59 & 4.35 \\
\hline & Mets & 7 & 5.00 & 7.00 & 7.50 & 10.00 & 10.54 & 14.00 \\
\hline \multirow{5}{*}{ 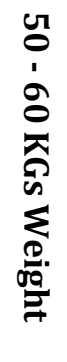 } & Time & 8 & 3.00 & 6.00 & 7.50 & 9.00 & 9.73 & 12.00 \\
\hline & $\mathbf{n}$ & 9 & 8 & 8 & 1 & 7 & 5 & 2 \\
\hline & K Cal & 10 & 7.13 & 18.29 & 25.88 & 41.91 & 46.89 & 70.34 \\
\hline & K Cal/ min & 11 & 2.37 & 3.04 & 3.45 & 4.65 & 4.81 & 5.86 \\
\hline & Mets & 12 & 5.00 & 7.00 & 8.50 & 10.00 & 10.72 & 14.00 \\
\hline \multirow{5}{*}{ 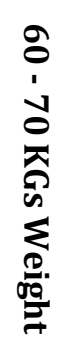 } & Time & 13 & 3.00 & 6.00 & 7.13 & 9.00 & 10.26 & 12.00 \\
\hline & $\mathbf{n}$ & 14 & 13 & 13 & 1 & 12 & 6 & 4 \\
\hline & K Cal & 15 & 8.29 & 21.15 & 28.37 & 46.83 & 57.15 & 80.06 \\
\hline & K Cal/ min & 16 & 2.76 & 3.52 & 3.97 & 5.20 & 5.57 & 3.71 \\
\hline & Mets & 17 & 5.00 & 7.00 & 8.10 & 10.00 & 11.06 & 14.00 \\
\hline \multirow{5}{*}{ 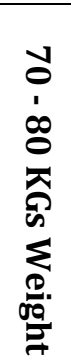 } & Time & 18 & 3.00 & 6.00 & 8.07 & 9.00 & & \\
\hline & $\mathbf{n}$ & 19 & 4 & 4 & 2 & 1 & & \\
\hline & K Cal & 20 & 9.26 & 23.61 & 37.41 & 57.65 & & \\
\hline & K Cal/ min & 21 & 3.08 & 3.93 & 4.63 & 6.40 & & \\
\hline & Mets & 22 & 5.04 & 7.00 & 9.10 & 10.00 & & \\
\hline
\end{tabular}


Graph-I Scatter Diagram Depicting Correlation Between Duration of Exercise and METS

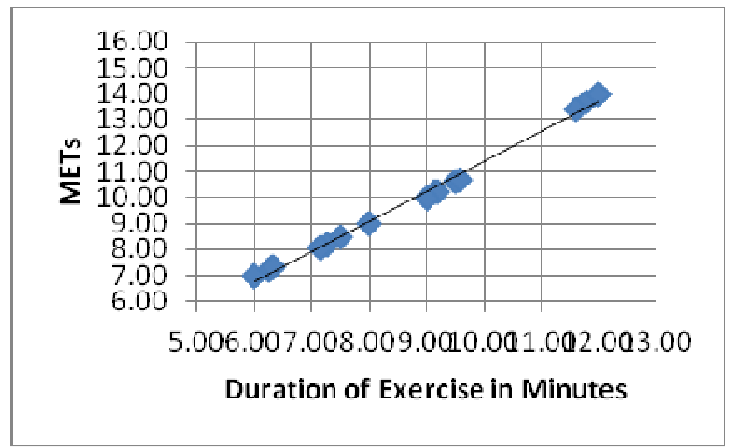

Graph-II Scatter Diagram Showing Correlation between Duration of Exercise and Work Output in Kcals

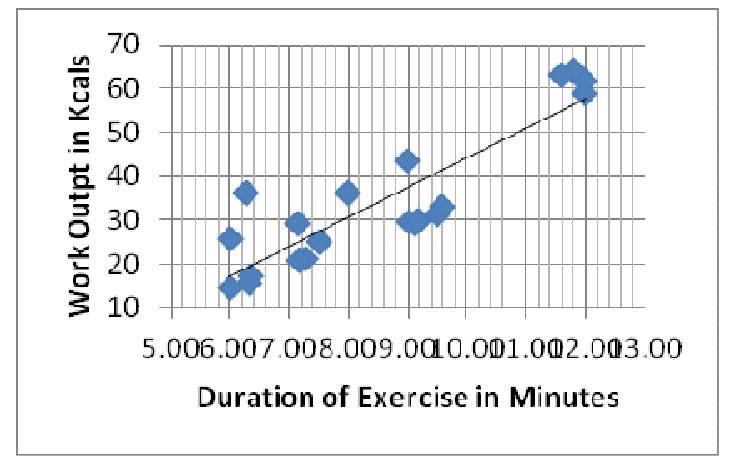

Graph-III Scatter Diagram Depicting Correlation between Body Mass in Kgs and METS

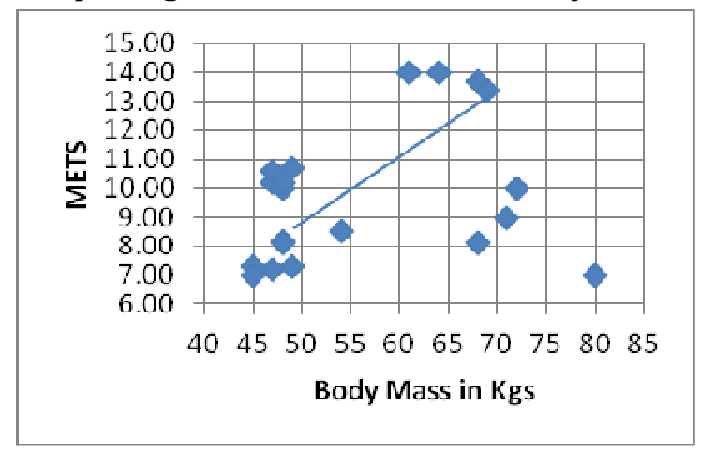

Graph-IV Scatter Diagram Shoing Correlation between Body Mass and Work Output

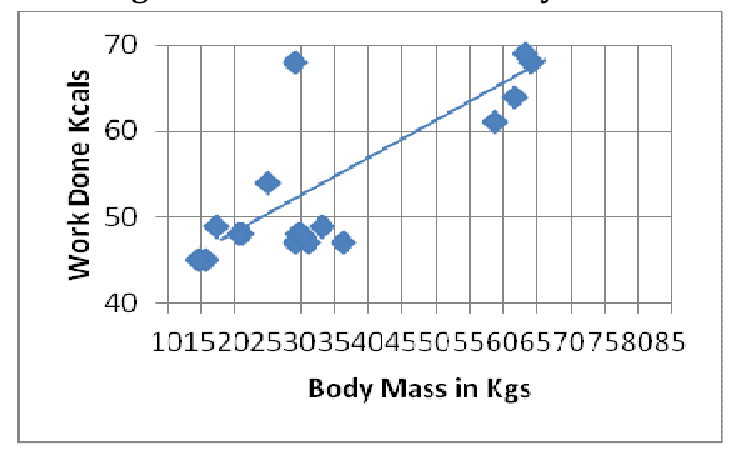


Graph-V Scatter Diagram Showing Correlation between Hemoglobin and Work Output Kcals

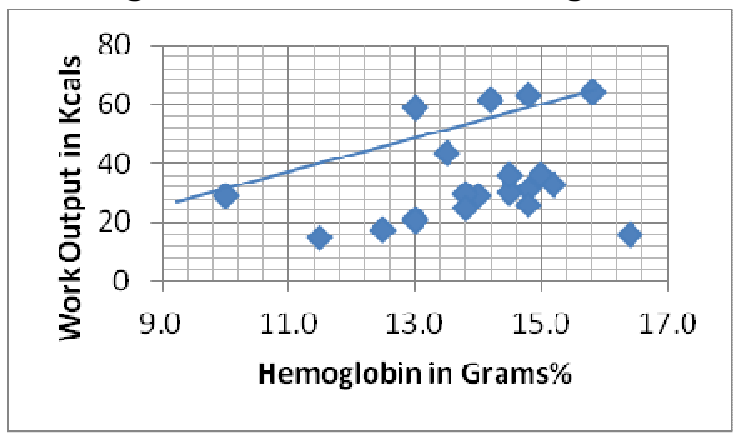

Graph-VI Line Plot Showing Relationship between Exercise duration Treadmill Belt Speed and METS

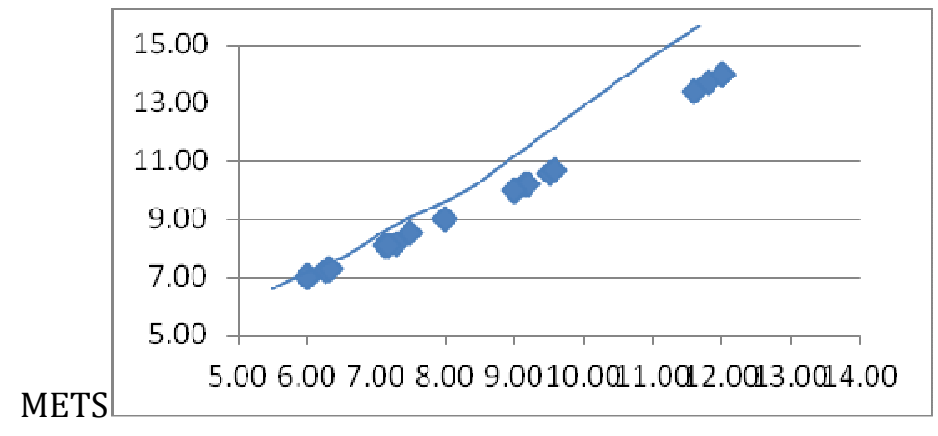

Duration in Minutes

RESULT: Table I presents physical data, energy expenditure and Mets Table II summarizes the essential individual profiles of total energy expenditure, and exercise duration in relation to their body mass and hemoglobin concentration.

To investigate if there is any pattern in the performance of individuals on Treadmill exercise according to their body mass , the values for the lowest to the highest weight ranges are presented in Table III.

Form Table III it is also evident that all the test subjects could not complete stage III and IV of the Bruce exercise protocol.

Graph I show a perfect correlation between the exercise duration of individuals and the corresponding values of METs.

Hence for any stage of the Bruce exercise protocol and duration of exercise the METs values are fixed do not depend on body mass.

Graph II shows relationship between duration of exercise and the quantity of work output on Kcals for all the test subjects.

When individual values of body mass are plotted against the corresponding values of METs the scatter in Graph III shows no evidence of correlation.

Graph IV shows correlation between body mass works out put

Graph V attempt to seek any possible correlation between individual's hemoglobin concentration and exercise performance.

Graph VI shows a linear relation for METs.

DISCUSSION: Quantification of work output for various routine day to day activities as well as sports events was attempted by several investigations and a energy cost list published. 
The present study attempts to estimate the energy cost of treadmill exercise through all its stages in a group of age matched healthy male medical student, differing though, in heights and weights. This gives a quantitative measure of stress.

Table I summarizes the essential features of each individuals work capacity stage wise and total, viewed against the corresponding figure of body mass, the hemoglobin concentration and heart rate response. For each stage, the fixed values of speed in KMPH, time limit, angle of inclination or passing through each stage is noted.

The customary practice to give a quantitative estimate of the energy cost of stress involved in treadmill exercise has been the use METs or metabolic equivalents. The MET is a unit of sitting/ resting Oxygen uptake ( $3.5 \mathrm{ml}$ of 02 per $\mathrm{kg}$ body weight per minute). The oxygen uptake during exercise when not directly estimated is often expressed in multiples of resting oxygen requirements (METs). The treadmill system is automated to give values of METs for each grade of activity computed from the speed, angle and duration that give a close approximation 02 uptake and reflect the work intensity/ load. Several studies correlate oxygen consumption to stress levels and oxygen uptake is shown to increase directly with work load. The maximal 02 consumption (VO2 Max) gives excellent estimates of subject's aerobic power and is an objective measure of exercise capacity. It defines the upper limits of cardiopulmonary system. But measurement of 02 uptake requires additional cost, time, requirement and potential discomfort to the patient raising the question of whether these measurements are justified in clinical practice. As a result predicting 02 uptake on treadmill in terms of metabolic equivalents (MET) based on belt speed, angle of inclination stage and duration of exercise is a common clinical practice. Hence for any given stage of exercise protocol and duration of exercise done, the values of METs are constant. This is amply illustrated in tables I and II. When statistical analysis for correlation between duration of exercise and METs is done a highly significant degree of correlation is established between these two parameters. This is clearly depicted in graph I scatter diagram, wherein correlation coefficient $₫$ is found to be 0.99 . The same fact is further illustrated graphically in graph VI, where METs values are shown to raise linearly and equally with the duration of exercise for the entire test.

In spite of widespread use of METs as a quantitative predictor of stress giving out predictions of 02 consumption in multiples of resting 02 uptake, such predictions can be very misleading. In several studies, wide differences were found between measured and predicted 02 uptake. The wide scatter around the regression line between predicted 02 uptake and exercise time or worked is well documented, yet poorly appreciated. Sullivan and associates found a higher correlation coefficient for measured 02 uptake than for predicted values. Hence reporting work in terms of the relatively unreliable measure; exercise time alone is fraught with inaccuracies. Therefore if quantifying work with precision is an important objective as in research studies, METs do not fulfill this purpose. Since they suffer the limitation of disregarding the influence of other variables such as body mass, age, gender etc., Direct studies of maximal $\mathrm{O}_{2}$ uptake demonstrate their role [1].

When age matched individuals of same gender are considered,. Variations in body mass assume added significance. It is estimated that $69 \%$ of the differences in $\mathrm{V} \mathrm{O}_{2}$ max scores among individuals can be explained simply by differences in body mass. Body mass is an important factor that affects the energy expended in many forms of exercise. That computation of METs does not reckon this important factor is illustrated when a relationship between these two variables is analyzed as in Scatter diagram (Graph) III. The wide scatter around the regression line and the correlation coefficient of 0.24 establish lack of correlation between body mass and 
METs. Howley E T et al estimated the caloric cost of running and walking one mile for men and women. Their data show that the energy cost for cross country running ranges between $8.2 \mathrm{Kcal}$ per minute for a $50 \mathrm{Kgs}$ person, to almost twice as much at $16.0 \mathrm{Kcal}$ for a person weighing 98 Kgs. In the present study when the energy cost is averaged for different weight ranges (Table III) for a 9 minutes completed exercise period, it is found to be $3.49 \mathrm{Kcal} / \mathrm{Min}$ for weight range of 40-50 Kgs, 4.65 Kcals/Min for 50-60 Kgs range $5.2 \mathrm{Kcal} / \mathrm{min}$ for 60-70 Kgs range and 6.4 $\mathrm{Kcal} / \mathrm{min}$ for $70-80 \mathrm{Kgs}$ range. It is apparent that the energy expenditure during exercise increased with body weight [2].

When the relation between body mass and work output are correlated statistically as shown in Graph IV, the correlation coefficient is found to be 0.6 which denotes significance. Therefore the role of this variable cannot be ignored in the computation of energetic of exercise. When similar relationship between body mass and METs are studied as in Graph III, there is wide scatter around regressions line and no significant relation could be established, since the correlation coefficient $r=0.24$. Clearly the predicted values of 02 usage as METs ignore the body mass factor but rely on duration of exercise in computation. There is a increasing overall energy expenditure with increasing body weights over a wide range from 40 to $70 \mathrm{Kgs}$, while it is apparently decreased for the highest weight range of 70-80 Kgs owing to the short duration of exercise they could sustain.

Instead of expressing the energy cost for a given body weight or weight ranges, if it is expressed in relation to unit body 'mass per minute, it could give a more standardized estimate and eliminates the individual differences. Accordingly Howley E.T et al from their studies of cross country running estimated that the energy cost averaged, $0.164 \mathrm{Kcal} / \mathrm{Kg} / \mathrm{min}$. By expressing energy cost in this manner, the differences between individuals are greatly reduced regardless of age, gender and body mass. In the present study the energy cost of Treadmill exercise for all the test subjects ranged between 0.05 to $0.08 \mathrm{Kcal} / \mathrm{Kg} / \mathrm{min}$ Tables presented depicting energy expenditure for various household, recreational and sports activities show comparable values. For example walking involved energy expenditure of $0.08 \mathrm{Kcal} / \mathrm{Kg} / \mathrm{min}$ and between 0.13 to $0.28 \mathrm{Kcal} / \mathrm{Kg} / \mathrm{min}$ for running depending on the speed of running taking into account wind resistance [2].

In another series of experiments, Ralston H.J demonstrated that the energy cost of walking on a Treadmill at $5.86 \mathrm{Km}$ per hour $(3.6 \mathrm{mph})$ and $2.93 \mathrm{Kms}$ per hour (1.8 $\mathrm{mph}$ ) was no different from normal walking on a hard surface at the same speeds. This indicates that people can generate essentially the same exercise stress either by walking on the level or walking at the same speed and distance on an exercise on treadmill. Such results also lend support to the use of laboratory data to quantify human energy expenditure in "real life" situations. But the fact remains that the total number of calories expended by a heavier person are considerably higher, than that expended by lighter counterpart, simply because the body mass must be transported during the activity and thus requires proportionately more energy.

In spite of the fact that energy expenditure or V 02 max increase with body mass, the apparent differences between individuals of same body mass could be ascribed to differences in body composition and Hemoglobin content. These two variables partly account for the gender differences in observed aerobic capacity; several studies demonstrate that V 02 max values for women are typically 15 to $30 \%$ below scores of men [3].

Lower body fat, relatively larger muscle mass and higher Hemoglobin provide the male with some advantage in aerobic power. Therefore a better expression of aerobic capacity or energy cost would be not in relation to body mass per se, but in relation to lean body mass. 
When expressed in relation to lean body mass, thus observed differences in energy expenditure between individuals of matching body mass and gender related differences narrow down. Fatty tissue being metabolically less active than muscle mass in exercise, the energy expenditure therefore correlates well with lean (fat free) body mass.

The influence of hemoglobin content in exercise performance is well known. Higher concentrations of hemoglobin enhance the Oxygen carrying capacity of blood and contribute favorably to exercise capacity and work output. Conversely low concentration of hemoglobin and low Oxygen carrying capacity limits exercise. In the present study the hemoglobin concentration of all the test subjects is also factored in evaluating the energy cost, and presented in the tables I and II. It is observed that the hemoglobin concentration of all the test subjects varied within a narrow range of 12 to $15 \mathrm{gms} \%$ with a mean value of $13.95 \pm 1.189$, since these subjects constitute of age and gender matched healthy homogeneous population. In the absence of wide differences in Hemoglobin concentration among test subjects, it is unlikely to explain the observed differences in work output. When the relationship between hemoglobin concentration and work output in Kcals is analyzed graphically (Graph V), this correlation coefficient is found to be 0.32 which implies no correlation in the present situation.

Bulk of the literature also correlates heart rate rise with $\mathrm{O}_{2}$ consumption, and establish a linear relationship between heart rate and Oxygen consumption and intensity of exercise. Although for each person's heart rate and Oxygen consumption time tends to be linear, the slope of the individual lines for different subjects differ considerably. Besides a number of other factors affecting heart rate dilute the accuracy of predicting $\mathrm{VO}_{2}$ max, the level of exercise intensity and limit its usefulness. The magnitude of heart rate rise in the present study varied widely from 20 to 100 beats/min among different test subjects with a mean of 58.85 beats per minute and a large standard deviation of \pm 20.55 as can be seen from table I. Heart rate monitoring however provides an objective measure of stress level that signals termination of test when certain target heart rate is reached as a safety precaution. The common practice is not to permit exercise to continue beyond the target heart rate which imposes a age related ceiling on hear rate rise, using this formula 220 - age. None of the test subjects in the present study could continue exercise to such a point of heart rate to be instructed to stop, since they discontinued well before.

Quantification of stress in various forms of physical activities, sports and laboratory exercise tests such as Treadmill exercise test help workout the economy of exercise. It is interesting to examine what difference it makes in energy cost when running at different speeds but covering the same distance. Because the relationship between $\mathrm{O}_{2}$ consumption and speed of running is linear, the caloric cost of running naturally increases with speed. But to cover a given distance at higher speed requires less time, and at a slower speed requires more time to be spent in running. In simple terms, if one runs a mile at speed of 10 miles/per hour, it require about twice as much energy per minute as running at 5 mile/hr. However the runner finishes the mile in 6 minutes, whereas running at slower speed requires twice the time or 12 minutes; consequently the energy cost of the mile is about the same. Therefore increasing or decreasing the speed (within the broad range of steady state paces) simply alters the length of the exercise period and has little effect on the total energy expended. Margara R. et al have worked out the energy cost for horizontal running and concluded that the net energy cost per Kg of body mass per kilometer traveled is approximately $1 \mathrm{Kcal} / \mathrm{Kg} / \mathrm{Km}$. Therefore the energy cost of locomotion is better related to distance than speed[4]. Comparing the cost of locomotion per unit distance 
traveled, it is well documented, that for both men and women, it is more costly to run than to walk a given distance. Speed Vs stage wise exercise duration are preprogrammed and fixed in the computerized Bruce protocol and hence the subjects have little choice as regards speed of run, but differ in exercise time they could endure. Viewed from this point, it is observed from the graph, the energy cost almost linearly increasing with speed and duration of exercise but differently for different weight ranges. For weight range of 40 to $50 \mathrm{Kgs}, 6.01 \mathrm{Kcal}$. For 3 minutes, 15.32 kcals for 6 minutes, 31.42 Kcals for 9 minutes. For weight range of 50 to 60 Kgs. 7.13 Kcals for 3 minutes, 18.29 Kcals for 6 mins and 41.91 Kcals for 9 mins. For the weight range of 60 to 70 Kgs. 8.29 Kcals for 3 mins, 21.15 Kcals for 6 mins and 46.83 Kcals for 9 mins. For 70 to $80 \mathrm{Kgs} .9 .26 \mathrm{Kcals}$ for 3 mins, $23.61 \mathrm{Kcals}$. for 6 mins and $57.65 \mathrm{Kcals}$ for 9 mins. These observations lend support to the role of not only exercise time but also body weight as important determinants of energy cost of exercise.

Another factor which is found to influence the energy cost, is the stride length and frequency, Daniels J18. et al 1978, made comparisons of running economy by determining steady state (02 consumption on a motorized treadmill). Their studies revealed that boys and girls were less economical in running compared to adults because they require 20 to $30 \%$ more Oxygen per unit body mass to run at particular speed. These differences have been attributed to greater stride frequency among children, as well as differences in mechanics that could contribute to the inferior movement economy[5]. The work of Pugh L.G.C.E. shows that running economy improves steadily throughout years 10 to 18. This in part explains the relatively poor performance of young children to distance running. Several studies point to shortening below or lengthening beyond a certain optimum stride length increased oxygen consumption and lowered economy. At least for well- trained runners, consensus of opinion recommends the stride length chosen by the runner himself as the optimum. This is in view of the fact that, seasoned runners have selected a particular stride length through years of practice and therefore this generally produces the most economical running performance blended to individual variations in body mass, inertia of limb segments and anatomic development. Biomechanical analysis may help the athletic to correct minor irregularities in movement patterns while running[6].

Similar study done by Venkataramana et al the quantification of training load was done by the time allocation pattern, combined with02 consumption, results indicated that there was a 1.3 fold of higher work rate with 02 consumption. The study clearly suggests the consideration of body weight component in the formulation of training program, is essential to achieve optimal sports performance[7].

CONCLUSION: One should appreciate that the utility value of quantification of exercise stress is enormous. It provides a scientific basis to access the absolute quantity of work, and individual can with stand on treadmill and to plan suitable dietary regimens, weight reduction programmes and to design ways of optimizing work rates and skill development and achieve economy of energy expenditure for a given task.

ACKNOWLEDGMENT: We extend our thanks to Ms G.Anusha, B.SC (Computer Science), Clerk in Department of Physiology, NRI Medical Collage, Sangivalasa, Visakhapatnam, Andhra Pradesh, India. 


\section{REFERENCES:}

1. Sullivan M. Genter F et al. The reproductively and Hemodynamic, Electrocardiographic and gas exchange data during treadmill exercise in patients with stable angina pectoris. Chest. 86:375-381,1984.

2. Howley E T ., and Glover M.E. The caloric cost of running and walking one mile in men and women. Medicine and science Sports. 6:235, 1974.

3. Ralstan H.J Comparison of energy expenditure during treadmill walking and floor walking. J.Appl. Physiol 15:1156, 1960.

4. Margara Ra,et al, Energy cost of running J.Appl. Physiol 18:367, 1963.

5. Daniels J. et al Differences and changes in VO2 among runners 10 to 18 years of age. Med.sci.sports 10:200, 1978.

6. Pugh L.G.C.E. Oxygen intake and treadmill running with observations of the effect of air resistance. J.Physiol 207:823, 1970.

7. Venkataramana Y, Surya kumari MVL, Sudhakara Rao S, and Balakrishna N, comparison of training loads and physiological responses in athletes; consideration of body weight implications. Journal of exercise physiology online. 3june 2004;7:134-139. 